\title{
FIGO Stage IC1 Ovarian Cancer
}

National Cancer Institute

\section{Source}

National Cancer Institute. FIGO Stage IC1 Ovarian Cancer. NCI Thesaurus. Code C128082.

Stage IC ovarian cancer with surgical spill. (FIGO, 2014) 